\title{
Trauma craneal no accidental en niños: costo económico directo de la atención médica en un hospital de tercer nivel
}

\author{
Arturo Loredo-Abdalá, ${ }^{1}$ Abigail Casas-Muñoz, ${ }^{1}$ Gabriel Alejandro González-Garay, ${ }^{2}$ \\ Raquel Ortiz-Hernández, ${ }^{3}$ Jessica María González-Corona ${ }^{1}$ y Leslie Viridiana Ramírez-Angoa ${ }^{1}$ \\ ${ }^{1}$ Coordinación de Estudios Avanzados sobre el Maltrato Infantil y su Prevención; ${ }^{2}$ Departamento de Metodología de la Investigación; ${ }^{3}$ Dirección de \\ Planeación. Secretaría de Salud, Instituto Nacional de Pediatría, Ciudad de México, México
}

\begin{abstract}
Resumen
Introducción: El trauma craneal no accidental (TCNA) es una forma extrema de abuso físico que se produce por la sacudida brusca de un lactante o preescolar. Objetivo: Describir el costo económico directo de la atención durante la hospitalización de 14 niños con diagnóstico confirmado de TCNA en un hospital pediátrico. Método: Análisis del costo de la enfermedad en pacientes con TCNA, atendidos entre 2001 y 2010. Se realizó análisis descriptivo y de sensibilidad. Se calculó costo económico directo parcial de la atención médica (días de estancia hospitalaria, exámenes de laboratorio y gabinete, procedimientos quirúrgicos y consultas por subespecialista), ajustado por la inflación, se tomó como año base 2001. Los pacientes se clasificaron en tres grupos: TCNA moderado, severo y fatal. Resultados: Los pacientes con TCNA severo generaron mayor costo en la atención médica ( $\$ 105794.88 \pm 33$ 201.91), en comparación con el grupo con TCNA moderado (\$37 $012.95 \pm$ 7154.87) y fatal (\$18 595.04 \pm 6424.47) ( $p<0.05)$. El costo total fue de 665467.98 pesos mexicanos (71 249.25 dólares internacionales). Conclusiones: El costo total de los 14 pacientes fue una cifra elevada como en otras partes del mundo. El costo económico directo se relaciona estrechamente con la gravedad del cuadro clínico.
\end{abstract}

PALABRAS CLAVE: Abuso infantil. Síndrome del bebé sacudido. Trauma craneal no accidental. Costos y análisis de costo. Economía médica.

\begin{abstract}
Introduction: Abusive head trauma (AHT) is an extreme form of physical abuse that is produced by abruptly shaking an infant or toddler. Objective: To describe the direct economic cost of care during hospitalization of 14 children with confirmed diagnosis of AHT in a pediatric hospital. Method: Analysis of the cost of disease in patients with AHT attended to between 2001 and 2010. Partial direct economic cost of medical care (days of hospital stay, laboratory tests and imaging studies, surgical procedures and subspecialist consultations) was calculated adjusting for inflation, with year 2001 taken as base year. Patients were classified in three groups (moderate, severe and fatal AHT). Descriptive and sensitivity analysis was carried out. Results: Patients with severe AHT generated higher medical care costs $(\$ 105,794.88 \pm 33,201.91)$ in comparison with the group of moderate $(\$ 37,012.95, \pm 7,154.87)$ and fatal $A H T(\$ 18,595.04 \pm 6424.47)(p<0.05)$. Total cost was $\$ 665,467.98$ Mexican pesos (\$71,249.25 international dollars). Conclusions: Total cost for the 14 patients was an elevated figure, as in other parts of the world. The direct economic cost is closely related to the severity of the clinical presentation.
\end{abstract}

KEY WORDS: Child abuse. Shaken baby syndrome. Abusive head trauma. Costs and cost analysis. Medical economics.

Correspondencia:

Abigail Casas-Muñoz

E-mail: abycas_md@ @otmail.com
Fecha de recepción: 07-05-2018

Fecha de aceptación: 28-08-2018

DOI: 10.24875/GMM.18004399
Gac Med Mex. 2018;154:671-680

Disponible en PubMed

www.gacetamedicademexico.com 


\section{Introducción}

El trauma craneal no accidental (TCNA) o shaken baby syndrome (SBS) es una forma extrema de abuso físico $^{1,2}$ que se produce por la sacudida brusca de un lactante o preescolar. Este mecanismo genera la rotación de la cabeza y movimientos de aceleración y desaceleración, que pueden causar la ruptura de los vasos comunicantes del cerebro (ocasionando hemorragias), hipoxia, daño cerebral difuso, daño en la columna cervical y en algunas estructuras del globo ocular. Se produce un traumatismo intracraneal que, en la mayoría de las ocasiones, no muestra evidencia externa en el cráneo que sugiera daño interno. Se manifiesta por una serie de alteraciones clínicas y patológicas que conforman un síndrome bien definido..$^{3,4}$ Existe una relación estrecha entre la fuerza del sacudimiento, el tiempo de duración de este y el grado de hipoxia que se genera. La hipoxia desencadena edema cerebral con elevación de la presión intracraneal, por lo que hay menor presión de perfusión sanguínea, produciendo isquemia cerebral y subsecuente daño axonal. Entre mayor hipoxia, mayor presencia de edema cerebral, determinante en la evolución clínica, que incrementa la gravedad y, por lo tanto, el costo de la atención médica. ${ }^{5,6}$

Entre los signos y síntomas más comunes que presentan estos pacientes se encuentran alteraciones del estado de conciencia (43 a $77 \%$ ), letargia (24 a $77 \%$ ), irritabilidad (7\%), crisis convulsivas (40 a $76 \%$ ), tono muscular aumentado o disminuido, vómito (15 a $24 \%$ ), rechazo al alimento (29\%), alteraciones de la respiración o apnea (34 a $41 \%$ ) y retardo o regresión en el desarrollo (12\%). Los hallazgos radiológicos en la tomografía axial computarizada de cráneo que más frecuentemente se encuentran son hemorragia subdural (29 a $80 \%$ ), hemorragia subaracnoidea (12 a $50 \%$ ), hemorragia intracerebral (8\%), hemorragia epidural (4 a $9 \%$ ), hemorragia 0 lesión parenquimatosa (9 a $37 \%$ ), edema cerebral (4 a $59 \%$ ), higromas (11 a $29 \%$ ). Además de las lesiones intracraneanas, se pueden encontrar fracturas en distintas partes del cuerpo (costales y huesos largos principalmente)..$^{4,-10}$ Aproximadamente la mitad de los pacientes con TCNA se presentan con deterioro severo, llegan inconscientes o moribundos. Estos pacientes se complican frecuentemente con hipotensión e hipoxemia. ${ }^{11}$ Se ha reportado que quienes sobreviven presentan secuelas importantes: entre 55 y $80 \%$ de los casos atrofia cerebral severa, así como déficit neurofuncional; los estudios de seguimiento se han realizado a cuatro o cinco años. La mortalidad se ha reportado en 19 a $35 \%$ de los casos, significativa si se considera que esta patología es $100 \%$ prevenible. ${ }^{3,7,10-13}$

La Academia Americana de Pediatría señala una incidencia de TCNA de 17 a $24 \%$ en Estados Unidos. ${ }^{3}$ En México no se ha podido precisar su frecuencia, básicamente porque no se establece el diagnóstico y cuando se instala, únicamente se hacen reportes aislados. ${ }^{12,13}$ A pesar de ello, las investigaciones sobre esta patología permiten conocer el cuadro clínico en el hogar y al ingreso en los servicios de urgencias, su fisiopatología, los servicios requeridos, así como la morbilidad y mortalidad..$^{14-19}$

Una faceta de esta patología que ha sido escasamente descrita a nivel internacional y no se ha descrito a nivel nacional es el impacto económico para la institución médica por su atención.

Para evaluar la carga económica de una enfermedad existen los estudios de costos de enfermedades, que identifican, cuantifican y valoran los recursos económicos asociados. Drummond ${ }^{20}$ reconoce la metodología utilizada para el cálculo de los costos de una enfermedad y considera que el costo de la enfermedad consta de tres componentes: costos directos, principalmente a cargo de la sistema de salud en el tratamiento de la enfermedad; costos indirectos, en términos de producción perdida debido a morbilidad o mortalidad prematura por la enfermedad; e intangibles, en términos de dolor, sufrimiento o reducción de la calidad de vida de quienes padecen la enfermedad. ${ }^{20}$

Existen pocos reportes específicamente sobre el costo de la atención de los pacientes víctimas de TCNA y los que se han generado provienen sobre todo de Estados Unidos, donde se ha estimado que el promedio del costo a lo largo de la vida de una víctima mortal de TCNA es de 5.7 millones de dólares (IC $95 \%$ = 3.2-9.2 millones de dólares). Para los pacientes que sobreviven, en 2010 se estimó que el costo promedio era de 257 millones de dólares en atención médica y costos directos relacionados. En este mismo año se estimó una incidencia de 4824 casos de TCNA. El costo a lo largo de la vida de estos casos fue de 13.5 billones (IC $95 \%=5.5-16.2$ billones de dólares), distribuidos en costo de la atención médica, 257 millones de dólares; costo de la educación especial, 552 millones de dólares; servicios de protección infantil o de justicia criminal, 322 millones de dólares; costo por pérdida de trabajo, 2 billones de 
dólares; por pérdida de calidad de vida, 10.3 billones de dólares. ${ }^{21,22}$

También se ha reportado cómo el costo de la atención médica de los pacientes con TCNA es más alto cuando se compara con el de pacientes con características sociodemográficas similares atendidos por otras causas, sobre todo porque requieren mayor tiempo de hospitalización, mayor uso de servicios médicos al egreso, así como mayor uso de medicamentos. ${ }^{23} \mathrm{En}$ lo que se refiere al costo por la atención en el servicio de urgencias y la hospitalización al momento del diagnóstico, en Estados Unidos se calculó que por atender a 6827 pacientes (IC $95 \%=6072-7582$ ) en urgencias y 12533 (IC $95 \%$ = 10 395-14 671) hospitalizaciones (28\% hospitalizados en el mismo hospital en el que se consultó el servicio de urgencias), el promedio de este costo fue de 2612 dólares por la atención en urgencias y 31901 dólares por la hospitalización. ${ }^{24}$

Hasta el momento no se ha descrito el costo económico que implica la atención específica de casos con el diagnóstico de TCNA en México y, por ende, no se conoce el impacto en al ámbito administrativo de un hospital pediátrico de tercer nivel de atención de nuestro país.

El objetivo de este estudio es describir el costo económico directo parcial de la atención durante la hospitalización de 14 pacientes con diagnóstico confirmado de trauma craneal no accidental en un hospital pediátrico de tercer nivel de la Ciudad de México, al momento del diagnóstico.

\section{Método}

Evaluación económica del tipo de análisis del costo de la enfermedad con un abordaje retrospectivo, bajo la perspectiva del sistema de cuidado de la salud que solo considera costos de la atención médica, ${ }^{25}$ en un periodo de 10 años, dada la baja frecuencia de detección de esta patología. El proyecto fue aprobado por el Comité de Investigación del Instituto Nacional de Pediatría, con número de registro 026/2016.

Se calculó el costo económico directo parcial de la atención médica mediante la revisión de fuentes secundarias: expedientes clínicos (físico y electrónico), expediente electrónico radiológico y de laboratorio. La población objetivo fueron los pacientes con diagnóstico confirmado de TCNA y por los que se solicitó interconsulta a la Clínica de Atención Integral al Niño Maltratado del Instituto Nacional de Pediatría (CAINM-INP) para su manejo, durante el periodo de 2001 a 2010.

\section{Definiciones operacionales y recopilación de datos}

El diagnóstico de TCNA se estableció por la presencia de hemorragia intracraneana y hemorragia retiniana, en conjunto con la valoración integral por el área médica, de enfermería, trabajo social y psicología de CAINM-INP en dicho periodo.

Los pacientes fueron clasificados de acuerdo con la gravedad del cuadro, con base en la presencia de edema cerebral y por la necesidad de ser atendidos en la unidad de terapia intensiva pediátrica (UTIP). De esta manera se clasificaron en tres grupos:

1. TCNA moderado, sin edema cerebral y que no requirió atención en UTIP.

2. TCNA severo, con edema cerebral por el que fue necesaria atención en la UTIP.

3. TCNA fatal, con edema cerebral y que causó el deceso (Tabla 1).

El costo de los recursos médicos utilizados se calculó con los valores de los costos del tabulador de cuotas de recuperación en nivel 6 del Instituto Nacional de Pediatría (INP), expresados en pesos mexicanos. Este tabulador en el nivel 6 considera el costo total de los servicios, tanto los directos como los indirectos y anualmente se ajusta de acuerdo con la inflación. Para su cálculo se emplean técnicas combinadas de macrocosteo y microcosteo ${ }^{26,27}$ El procedimiento de cálculo de estas cuotas está apegado a la Ley de Ingresos de la Federación y esta a su vez a la Ley General de Salud. ${ }^{26}$ El costo de las complicaciones más inmediatas (otros diagnósticos que se desarrollan por la severidad del cuadro) relacionadas con el TCNA y que forman parte de la atención del TCNA está incluido en este cálculo.

Se utilizó el mismo procedimiento para calcular el costo que los pacientes pagaron a la institución de acuerdo con su nivel asignado.

Para establecer el costo por la estancia en los diferentes servicios se utilizó el costo por día-cama, en el que se consideran los costos directos durante la hospitalización de los pacientes y ello incluye medicamentos, curaciones, procedimientos, la intervención de los médicos residentes y el personal de enfermería, excepto la alimentación de los niños. ${ }^{28}$

Debido a que en esta evaluación económica se incluyeron pacientes atendidos en diferentes años, se pasaron los valores de los costos de las unidades monetarias nominales (corrientes) a reales (constantes), para eliminar el efecto producido por la inflación (se deflactaron). Para llevar a cabo la deflactación del valor del costo de 
Tabla 1. Características de 14 pacientes con diagnóstico de trauma craneal no accidental (TCNA) durante su hospitalización, al momento del diagnóstico, y secuelas que se identificaron durante la hospitalización

\begin{tabular}{|c|c|c|c|c|c|c|c|c|}
\hline \multirow[t]{2}{*}{ Característica } & \multicolumn{2}{|c|}{$\begin{array}{l}\text { TCNA moderado } \\
(\mathrm{n}=4)\end{array}$} & \multicolumn{2}{|c|}{$\begin{array}{l}\text { TCNA grave } \\
(n=4)\end{array}$} & \multicolumn{2}{|c|}{$\begin{array}{l}\text { TCNA fatal } \\
(\mathrm{n}=6)\end{array}$} & \multicolumn{2}{|c|}{$\begin{array}{l}\text { Total } \\
(n=14)\end{array}$} \\
\hline & $\mathbf{n}$ & $\%$ & n & $\%$ & $\mathbf{n}$ & $\%$ & $\mathrm{n}$ & $\%$ \\
\hline \multicolumn{9}{|c|}{ Principales características al ingreso que se utilizaron para clasificar la severidad del TCNA } \\
\hline $\begin{array}{l}\text { Presentó alteración del estado de conciencia al } \\
\text { ingreso }\end{array}$ & 1 & 25 & 4 & 100 & 5 & 83 & 10 & 71 \\
\hline Requirió intubación & 1 & 25 & 4 & 100 & 5 & 83 & 10 & 71 \\
\hline Presencia de edema cerebral en la TAC de cráneo & 0 & 0 & 4 & 100 & 6 & 100 & 10 & 71 \\
\hline Requirió atención en UTIP & 0 & 0 & 4 & 100 & 0 & 0 & 4 & 29 \\
\hline \multicolumn{9}{|l|}{ Secuelas identificadas durante la hospitalización } \\
\hline Secuelas motoras & 3 & 75 & 4 & 100 & & NV & 7 & 50 \\
\hline Secuelas visuales & 3 & 75 & 4 & 100 & & NV & 7 & 50 \\
\hline Secuelas auditivas & 0 & 0 & 4 & 100 & & NV & 4 & 29 \\
\hline Secuelas en el lenguaje & 4 & 100 & 3 & 75 & & NV & 7 & 50 \\
\hline Persistencia de crisis convulsivas & 2 & 50 & 4 & 100 & & NV & 6 & 43 \\
\hline Presentó retardo global del desarrollo & 2 & 50 & 4 & 100 & & NV & 6 & 43 \\
\hline
\end{tabular}

Mediana Mín.-máx. Mediana Mín.-máx. Mediana Mín.-máx. Mediana Mín.-máx.

\begin{tabular}{lllllllll}
\hline Edad en meses & 5 & $4-8$ & 7.5 & $2-9$ & 5 & $2-14$ & 6 & $2-14$
\end{tabular}

TCNA = trauma craneal no accidental, UTIP = unidad de terapia intensiva pediátrica, NV = no valorable.

cada recurso utilizado anualmente por paciente, se calculó el coeficiente deflactor anual (Figura 1) y se tomaron como base los valores del tabulador de cuotas de recuperación del INP del año 2001. Ello dio como resultado el valor real (Figura 2).

\section{Procedimiento de cálculo de los costos}

Mediante el abordaje down-up ("abajo hacia arriba") ${ }^{25}$ se identificaron los generadores de costo relacionados directamente con la atención médica: días de estancia hospitalaria, número de consultas por especialista, procedimientos quirúrgicos y exámenes de laboratorio y de gabinete efectuados. Se estimó el costo de cada componente por separado y luego se sumaron para obtener el costo total. ${ }^{29} \mathrm{El}$ análisis de costos de la enfermedad se realizó en dos etapas: en la primera se cuantificaron los recursos médicos utilizados generadores del costo (Tabla 2). En la segunda se determinaron los valores del costo unitario correspondiente a cada recurso. Para ello, primero se obtuvo el valor nominal y posteriormente el valor real (deflactado).

Los costos médicos directos se estimaron ${ }^{30}$ multiplicando el valor real de cada recurso médico utilizado por su frecuencia de uso (Figura 3). Los costos totales y por grupo se calcularon en pesos mexicanos, posteriormente se convirtieron a dólares internaciones (unidad monetaria dólar Geary-Khamis), para lo cual se utilizó el costo de equivalencia de 9.34 pesos mexicanos del 31 de diciembre de 2001 (año base para el cálculo en pesos mexicanos), emitido por el Banco Mundial. ${ }^{31}$

\section{Análisis estadístico}

El análisis de los datos se llevó a cabo con el software SPSS versión 21.

Se realizó un análisis estadístico descriptivo para la muestra total y por grupos de análisis. Se calcularon frecuencias y porcentajes para las variables de las características de los pacientes. Para la edad y la cantidad de recursos utilizados se empleó mediana, mínimo y máximo. Para los costos se calculó media y desviación estándar, así como mediana, mínimo y máximo (debido a la distribución de los datos).

Para identificar diferencias significativas entre los grupos y contrastar la hipótesis de igualdad entre ellos, se realizó la prueba no parámetrica de Kruskal-Wallis, se consideró como estadísticamente significativo un 


$$
\text { Coef } . \text { deflactor }=\frac{I P C_{f}}{I P C_{l}}
$$

Donde:

Coef. deflactor $=$ coeficiente deflactor.

$\mathrm{IPC}_{\mathrm{f}}=$ índice de precios de consumo final (se utilizaron los IPC de los años en que se atendieron los pacientes).

IPCi = índice de precios de consumo inicial (se utilizó el IPC de 2001).

Figura 1. Cálculo del coeficiente deflactor. ${ }^{28}$

$$
V R=\frac{I N}{\text { Coef.deflactor }}
$$

Donde:

$\mathrm{VR}=$ valor real.

IN = importe nominal.

Coef. deflactor $=$ coeficiente deflactor .

Figura 2. Cálculo de la deflactación.

$$
C T_{j k w} \Sigma_{i=1}^{n} Q R_{j k i}{ }^{*} P R_{j k i}-
$$

Donde:

CT = costo total de la atención para el evento $j$, para el individuo $k$, con la condición de salud w.

$\mathrm{QR}=$ cantidad utilizada del recurso $i$ para la atención médica de un evento médico $j$ del paciente $k$ con la condición de salud $w$.

$\mathrm{PR}=$ precio o costo unitario del recurso $i$.

$I=$ recursos utilizados para la realización de la atención médica $\{1,2 \ldots . . . n\}$.

$J=$ tipo de recurso médico (1. Días de estancia hospitalaria, 2. Número de consultas por especialista, 3. Procedimientos quirúrgicos realizados, 4. Exámenes de laboratorio y 5. Exámenes de gabinete realizados). $k=$ Pacientes $\{1,2 \ldots n\}$.

Figura 3. Cálculo de la deflactación.

valor de $p<0.05$. Para el análisis de sensibilidad se llevó a cabo el cálculo por los estratos de severidad (TCNA moderado, severo y fatal).

\section{Resultados}

Se identificaron 14 pacientes con el diagnóstico confirmado de TCNA, 10 (71\%) fueron del sexo masculino; la mediana de la edad fue de seis meses (mínimo dos y máximo 14); seis pacientes (43\%) fallecieron durante su estancia en urgencias, la mediana de tiempo de estancia hospitalaria fue de 22 días (mínimo dos, máximo 129). Otras características de los pacientes se describen en la Tabla 1.

Los casos con TCNA severo representaron un costo promedio total de la atención médica mayor (105 794.88 \pm 33201.91 pesos mexicanos) en comparación con el grupo con TCNA moderado (37 $012.95 \pm 7154.87$ ) y con el grupo con TCNA fatal (18 595.04 \pm 6424.47$)(p<0.01)$.

El desglose del costo de cada recurso se muestra en la Tabla 3. El costo en dólares internacionales se muestra en la Tabla 4.

El costo por la estancia hospitalaria también fue más alto en el grupo con TCNA severo (52 $803.15 \pm$ 29633.69 pesos mexicanos) ( $<<0.01$ ), que en los pacientes con TCNA moderado (22 $650.00 \pm 6786.35)$ y el grupo con TCNA fatal, cuyo costo fue el más bajo $(4174.55 \pm 2421.21)$ ya que su estancia hospitalaria fue menor, con una mediana de 2.5 días (0-11).

Respecto a los exámenes de laboratorio, no hubo diferencia ( $p>0.05)$ entre los costos en los pacientes: TCNA moderado, $892.05 \pm 397.59$ pesos mexicanos; TCNA severo, $23517.73 \pm 9465.91$ y TCNA fatal, $4752.71 \pm 3270.27$.

Los costos por los exámenes de gabinete tampoco tuvieron diferencias $(p>0.05)$ entre los tres grupos: TCNA moderado, $7175.56 \pm 4953.61$ pesos mexicanos; TCNA severo, $10040.05 \pm 6285.74$ y TCNA fatal, $5372.96 \pm 2294.97$.

En los procedimientos quirúrgicos el costo tampoco fue diferente $(p>0.05)$, entre los grupos: TCNA severo, $16327.77 \pm 12522$ pesos mexicanos; TCNA moderado, $3378.32 \pm 3996.57$ y TCNA fatal, $242.68 \pm$ 2626.69 .

El costo por las interconsultas a los diferentes subespecialistas tampoco mostró diferencias entre los grupos: $2911.96 \pm 657.94$ pesos mexicanos en TCNA moderado, $3105.73 \pm 692.99$ en TCNA severo y $1490.36 \pm 725.69$ en TCNA fatal.

El costo total por la atención de los 14 pacientes fue de 665467.98 pesos mexicanos (71 249.25 dólares internacionales). El valor que se pagó por la atención de los pacientes a la institución fue de 65360.58 pesos mexicanos, lo que representa que se subsidió $90.17 \%$ (600 107.4 pesos mexicanos) del costo de la atención.

El análisis de sensibilidad mostró una diferencia $(p<0.01)$ en el costo total de la atención entre los diferentes estratos de severidad (Figura 4). 
Tabla 2. Cantidad de recursos utilizados en la atención médica en 14 pacientes con diagnóstico de trauma craneal no accidental durante su hospitalización, al momento del diagnóstico

\begin{tabular}{|c|c|c|c|c|c|c|c|c|}
\hline \multirow[t]{2}{*}{ Recurso que generó costo } & \multicolumn{2}{|c|}{ TCNA moderado } & \multicolumn{2}{|c|}{ TCNA grave } & \multicolumn{2}{|c|}{ TCNA fatal } & \multicolumn{2}{|c|}{ Total } \\
\hline & Mediana & Mín.-máx. & Mediana & Mín.-máx. & Mediana & Mín.-máx. & Mediana & Mín.-máx. \\
\hline Días de estancia en urgencias & 0.50 & $0-5$ & 1 & $0-11$ & 5 & $2-9$ & 2.5 & $0-11$ \\
\hline Días de estancia en piso & 29.5 & $13-31$ & 34 & $20-117$ & 0 & 0 & 16.5 & $0-117$ \\
\hline Días de estancia en UTIP & 0 & 0 & 13 & $1-25$ & 0 & 0 & 0 & $0-25$ \\
\hline Cantidad de exámenes de laboratorio & 4.5 & $3-8$ & 67 & $59-81$ & 16 & $5-34$ & 16 & $3-81$ \\
\hline Cantidad de exámenes de gabinete & 7.5 & $5-11$ & 9 & $6-17$ & 7.5 & $4-11$ & 8.5 & $4-17$ \\
\hline Cantidad de procedimientos quirúrgicos & 0.5 & $0-1$ & 1.5 & $0-4$ & 0.5 & $0-1$ & 1 & $0-4$ \\
\hline Cantidad de interconsultas a subespecialidades & 7 & $5-9$ & 7 & $7-10$ & 5.5 & $4-8$ & 7 & $4-10$ \\
\hline
\end{tabular}

TCNA = trauma craneal no accidental, UTIP = unidad de terapia intensiva pediátrica.

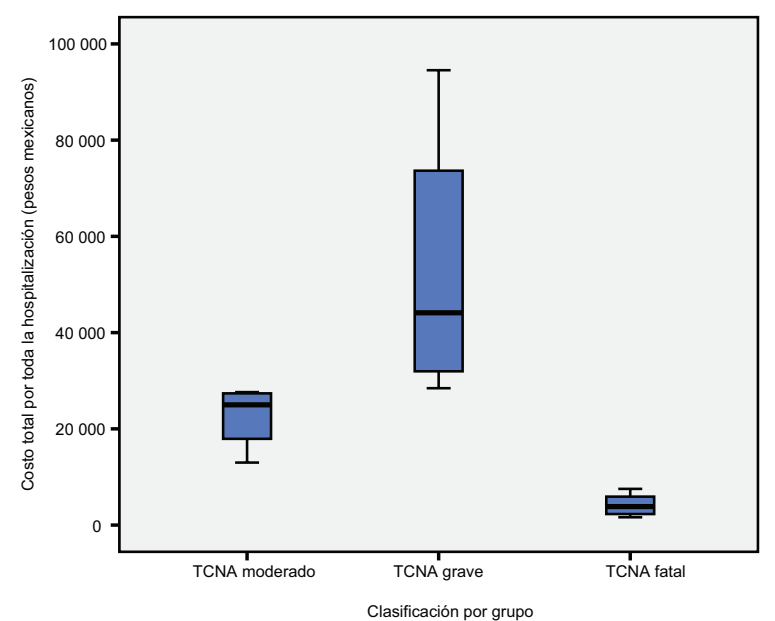

Figura 4. Análisis de sensibilidad (prueba de Kruskal-Walis con una $p<0.05)$.

\section{Discusión}

En este estudio se muestran de manera desglosada los costos directos parciales parciales de la atención médica que implica la atención de los pacientes con TCNA, a diferencia de los análisis hasta el momento referidos (que los describen de manera global). Este desglose incluyó la cantidad días de estancia hospitalaria, los procedimientos quirúrgicos requeridos, los diferentes estudios de laboratorio y gabinete y su cantidad en estos pacientes, así como el número de especialistas en medicina crítica, en neurocirugía, principalmente, cuya intervención es necesaria. Ello explica por qué el costo de la atención médica y quirúrgica es tan elevado. ${ }^{21-24}$

Tomando en cuenta la severidad del cuadro clínico de estos pacientes, se explica la hospitalización prolongada, la necesidad de que sean atendidos en los servicios de medicina crítica pediátrica (urgencias y terapia intensiva) y que la mayoría de ellos requiere al menos un procedimiento quirúrgico, en lo que coincidimos con Miller y Peterson..$^{22,24}$ En los niños que sobrevivieron, la severidad del cuadro determinó la duración de la estancia hospitalaria, mayor en los niños con TCNA severo.

En estos pacientes, el establecimiento y vigilancia de la condición metabólica e infecciones intrahospitalaria explica la cantidad de exámenes de laboratorio, lo que genera un costo alto, principalmente cuando son atendidos en la UTIP. De los estudios de gabinete, destacó la realización de 32 tomografías de cráneo, 32 radiografías de diferentes partes del cuerpo y ultrasonidos diversos. Todos los estudios fueron indispensables para precisar la condición neurológica, ocular y ósea de los pacientes, así como la modalidad de la agresión.

Los niños fueron sometidos a diversos procedimientos quirúrgicos, principalmente las intervenciones en cráneo, para resolver la existencia de fracturas hundidas, hematomas e higromas. ${ }^{24}$ La cantidad de especialistas que intervinieron en la atención de estos pacientes fue importante (en promedio siete por paciente), lo que elevó el costo de su atención.

La investigación de los casos con TCNA en nuestro medio se ha orientado hacia el conocimiento específico de las características fundamentales en el campo médico pediátrico, en el social y legal. En el área clínica se han descrito los factores desencadenantes del TCNA, la severidad del cuadro clínico, los estudios requeridos para precisar el diagnóstico y el manejo médico-social-legal en cada paciente. ${ }^{10}$

Una faceta de esta modalidad de maltrato infantil estudiada en otros países es la referente a precisar el costo requerido para el manejo integral de los pacientes víctimas de TCNA. ${ }^{23,32-34}$ Los costos iniciales 


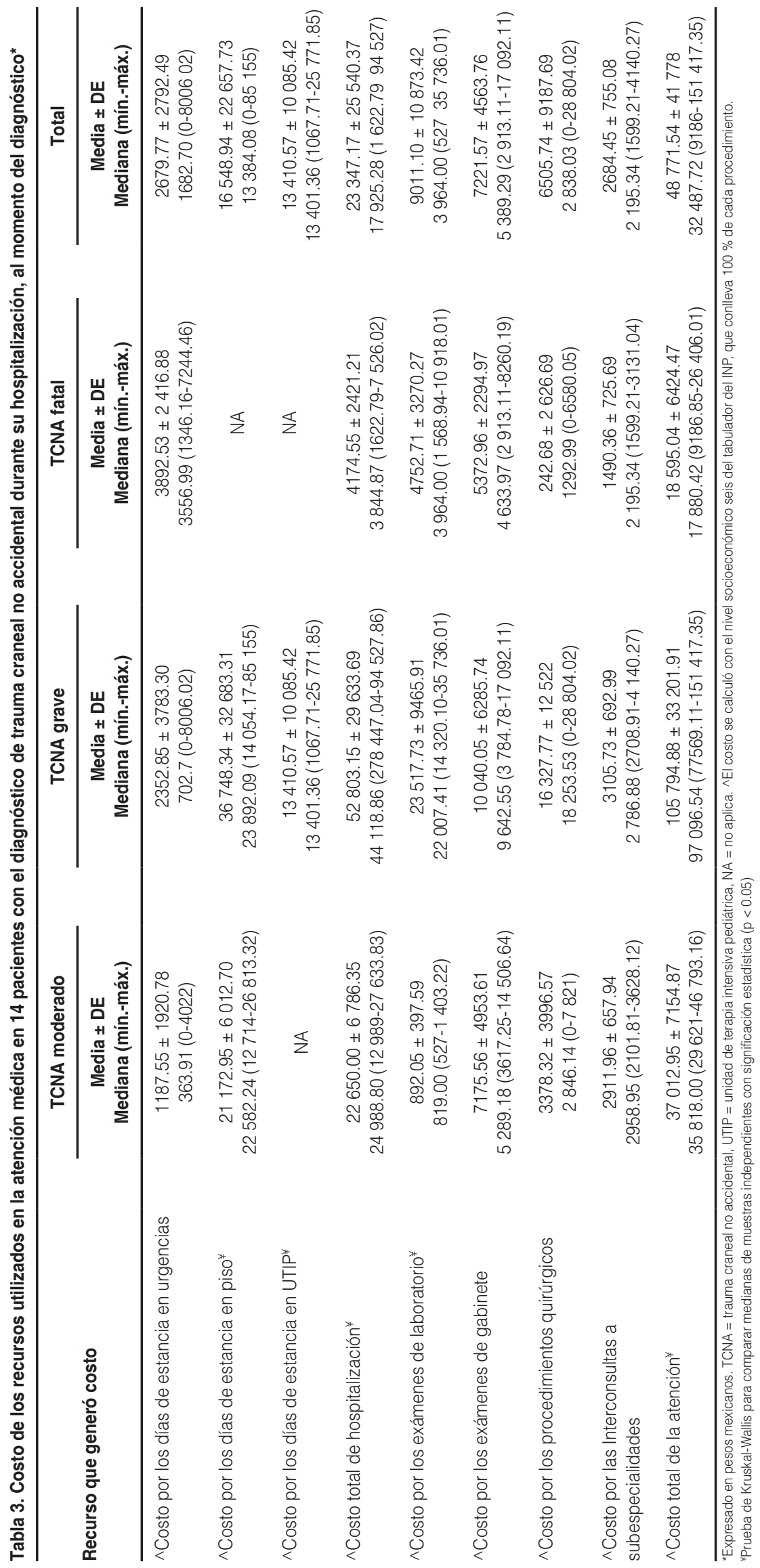




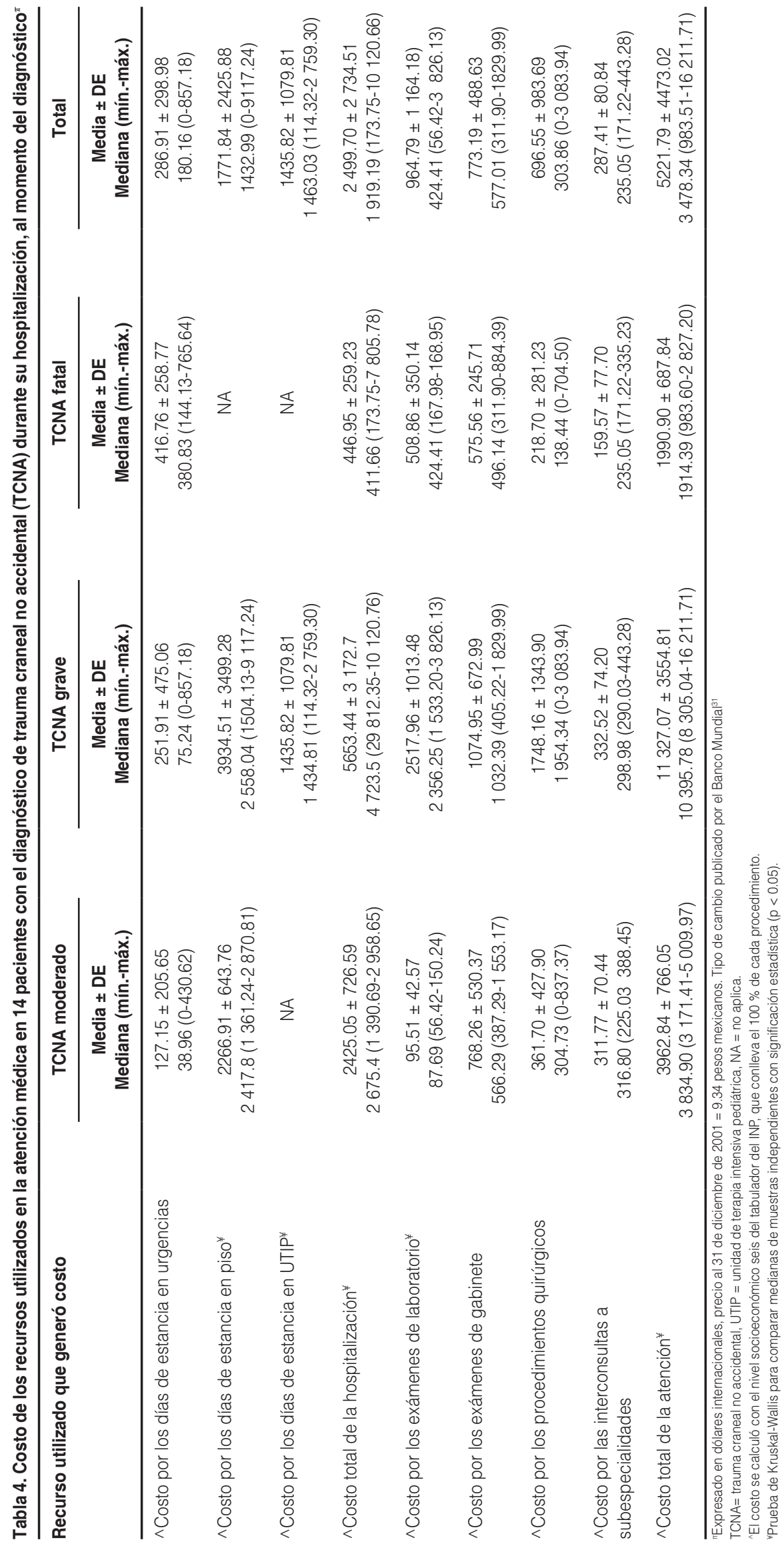


de hospitalización reportados por algunos investigadores varían de 18000 a 70000 dólares por niño y los costos médicos promedio pueden superar los 300000 dólares por niño. ${ }^{35}$ Los costos médicos y administrativos requeridos para la atención a largo plazo superan los 300000 y 1000000 dólares por caso, respectivamente. ${ }^{36}$ En México no se había realizado un estudio que señalara el costo directo de la atención médica de pacientes con TCNA en un centro hospitalario de tercer nivel de atención pediátrica.

La severidad del cuadro clínico de esta modalidad de abuso físico requiere una atención en tres áreas específicas de hospitalización (urgencias, UTIP y piso), la intervención de médicos con diversas especialidades y la realización de estudios de laboratorio y de gabinete muy específicos; por lo tanto, solo instituciones hospitalarias pediátricas de tercer nivel de atención pueden brindar la atención integral que este tipo de pacientes requieren, con el consiguiente impacto económico para la institución. A lo anterior hay que agregar que si estos niños sobreviven, tienen secuelas neurológicas, visuales, auditivas y osteoarticulares de grado y severidad variables que requieren atención médica y paramédica especializada por un periodo prolongado, con la consiguiente carga económica para la familia y el sector salud. Aunque existen estudios en otros países en este sentido, ninguno llena completamente los costos específicos de una atención completa inmediata y a largo plazo, ya que en general se analizan una o varias situaciones del maltrato infantil. ${ }^{23,37}$

Los costos que se han podido establecer en este estudio permiten demostrar que el gasto es bastante elevado para nuestro medio, tal como ocurre en otras partes del mundo, donde se establece el costo en dólares. Por ello, insistimos en la pertinencia de apoyar al máximo el desarrollo de programas preventivos del maltrato infantil y específicamente del TCNA, ya que seguramente será más redituable su aplicación que invertir en la atención y corrección de la morbimortalidad de estos pacientes.

\section{Limitaciones del estudio}

Como se mencionó en la sección de métodos, el costo de la atención médica que se computó para estos pacientes incluyó las complicaciones más inmediatas (otros diagnósticos), las cuales no fueron calculadas por separado y el costo de estos diagnósticos fue atribuido al diagnóstico de base (TCNA).
Debido a que no fue posible disponer de información uniforme sobre el seguimiento de estos pacientes en las fuentes de información consultadas, se realizó un estudio transversal, razón por la que no se logró establecer el costo a largo plazo. Es recomendable realizar un análisis que incluya el seguimiento para establecer la probabilidad de años de supervivencia y el costo de la atención de las secuelas.

Los costos calculados no reflejan el costo nacional porque se utilizó el tabulador de cuotas de recuperación de un hospital de tercer nivel de atención como el Instituto Nacional de Pediatría. Aunque es pertinente aclarar que habitualmente este tipo de pacientes se atienden en instituciones de tercer nivel, por la gravedad con la que llegan al momento de solicitar atención médica.

El análisis completo del costo de una enfermedad idealmente debe incluir costos directos, indirectos e intangibles; sin embargo, la presente investigación se realizó con un enfoque de prevalencia y no de incidencia, ${ }^{38,39}$ el cual incluye la estimación de costos de por vida. Se sugiere llevar a cabo este tipo de análisis para completar el panorama económico que representa esta patología, considerando que en las últimas décadas se han incremento los costos de la producción de los servicios de salud, tanto en cifras absolutas per cápita, como en fracción del producto interno bruto y en relación con la inflación. ${ }^{40}$

\section{Conclusiones}

El costo económico directo de la atención hospitalaria de los 14 pacientes con diagnóstico de TCNA fue alto para nuestro medio, aunque no es comparable con los reportados en Estado Unidos.

En término generales se relaciona estrechamente con la gravedad del cuadro clínico, que conlleva una estancia hospitalaria prolongada, sobre todo en los servicios de medicina crítica (urgencias y terapia intensiva), un volumen importante de exámenes de laboratorio y gabinete y la participación de diversos especialistas para su atención.

Se insiste en la necesidad de desarrollar programas preventivos, considerando que esta patología es prevenible en $100 \%$ de los casos.

\section{Agradecimientos}

Agradecemos a los médicos pasantes de servicio social en medicina María Teresa Arias González y Víctor Alam Gaona Badillo, así como de administración 
Julio César Huerta Navarro, su participación en la revisión de los tabuladores de cuotas de recuperación por año y en el cálculo de los precios reales de cada servicio, así como en la búsqueda de bibliografía.

\section{Bibliografía}

1. Caffey J. The whiplash shaken infant syndrome: manual shaking by the extremities with whiplash-induced intracranial and intraocular bleedings, linked with residual permanent brain damage and mental retardation. Pediatrics. 1974:54:396- 403.

2. Harding B, Risdon RA, Krous HF. Shaken baby syndrome. BMJ. 2004;328:720-721.

3. American Academy of Pediatrics: Committee on Child Abuse and Neglect. Shaken baby syndrome, rotational cranial injuries: technical report. Pediatrics. 2001;108:206-210.

4. Karibe H, Kameyama M, Hayashi T, Narisawa A, Tominaga T. Acute subdural hematoma in infants with abusive head trauma: a literature review. Neurol Med Chir (Tokyo). 2016;56:264-273.

5. Rufo-Campos M. El síndrome del niño sacudido. Cuad Med Forense. 2006;12:39-45

6. Mian M, Shah J, Dalpiaz A, Schwamb R, Miao Y, Warren K, et al. Shaken baby syndrome: a review. Fetal Pediatr Pathol. 2015;34:169-175.

7. King JW, MacKay M, Sirnick A, Canadian Shaken Baby Study Group. Shaken baby syndrome in Canada: clinical characteristics and outcomes of hospital cases. CMAJ. 2003;168:155-159.

8. Sieswerda-Hoogendoorn T, Boos S, Spivack B, Bilo RA, Van-Rijn RR. Educational paper: abusive Head Trauma Part I. Clinical aspects. Eur J Pediatr, 2012;171:415-423,

9. Bhardwaj G, Chowdhury V, Jacobs MB, Moran KT, Martin FJ, Coroneo MT. A systematic review of the diagnostic accuracy of ocular signs in pediatric abusive head trauma. Ophthalmology. 2010;117:983-992.

10. Loredo-Abdalá A, Casas-Muñoz A, Trejo-Hernández J, Melquiades-Para I, Martín-Martín V. Síndrome del niño sacudido: cuadro clínico y evolución de 17 casos en el Instituto Nacional de Pediatría. Acta Pediatr Mex. 2015;36:72-80.

11. Han BK, Towbin RB, De-Courten-Myers G, McLaurin RL, Ball WS. Reversal sign on CT: effect of anoxic/ischemic cerebral injury in children AJNR Am J Roentgenol. 1990:154:361-368.

12. Perea-Martínez A, Loredo-Abdalá A, Guicho-Alba E. El niño sacudido-impactado una modalidad del maltrato físico severo. En: Loredo-Abdalá $A$ Maltrato en niños y adolescentes. México: Editores de Textos Mexicanos; 2004.

13. Delgado FA, Lavalle VA, Torres GS. Síndrome del niño sacudido. Bol Med Hosp Infant Mex. 1995;52:481-484.

14. Block R, Christian CW, Committee Child Abuse and Neglect, American Academy of Pediatrics. Abusive head trauma in infant and children. Pediatrics. 2009;123:1409-1411.

15. Chiesa A, Dunhaime AC. Traumatismo craneoencefálico por maltrato. Pediatr Clin N Amer. 2009;56:317-331.

16. Guthkelch AN. Infantile subdural hematoma and its relationship to whiplash injuries. Br Med J. 1971:2:430-431.

17. Caffey J. Multiple fractures in the long bones of infants suffering from chronic subdural hematoma. Am J Roentgenol Radium Ther. 1946;56:163-173.

18. Silverman FN. The roentgen manifestations of unrecognized skeletal trauma in infants. Am $\mathrm{J}$ Roentgenol Radium Ther Nucl Med. 1953;69:413-427.
19. Ommaya AK, Faas F, Yamell $P$. Whiplash injury and brain damage: an experimental study. JAMA. 1968;204:285-289.

20. Drummond M. Cost-of-illness studies: a major headache? Pharmacoeconomics. 1992;2:1-4

21. Brown DS, Fang X, Florence CS. Medical costs attributable to child maltreatment: a systematic review of short-and long-term effects. Am J Prev Med. 2011;41:627-635.

22. Miller TR, Steinbeigle R, Lawrence BA, Peterson C, Florence C, Barr M, et al. Lifetime cost of abusive head trauma at ages 0-4, USA. Prev Sci. 2017;6:1-10.

23. Peterson C, Xu L, Florence C, Parks SE, Miller TR, Barr RG, et al. The medical cost of abusive head trauma in the United States. Pediatrics. 2014;134:91-99.

24. Peterson C, Xu L, Florence C, Parks SE. Annual cost of U.S. hospital visits for pediatric abusive head trauma. Child Maltreat. 2015;20:162-169.

25. Jo C. Cost-of-illness studies: concepts, scopes, and methods. Clin Mol Hepatol. 2014;20:237-337.

26. Secretaría de Salud. Manual institucional y guía sectorial para la aplicación de la metodología de costos. México: Secretaría de Salud; 2011.

27. Instituto Nacional de Pediatría. Procedimiento para la actualización de cuotas de recuperación. En: Manual de procedimientos de la Dirección de Planeación. México: Instituto Nacional de Pediatría; 2011.

28. Yirepa, Finanzas Básicas. [Sitio web]. Deflactar. Disponible en: http:// yirepa.es/deflactar.html.

29. Baeza-Cruz G, Peniche-Otero G, Alva-Esqueda ME, Naranjo-Muedano M, Soria-Suárez N, Morales-Flores HJ. Análisis de costo de la enfermedad, del tratamiento, las complicaciones e intervenciones de la hipercolesterolemia en México en 2016. Value Health Reg Issues. 2018;17:56-63.

30. Tarricone R. Cost-of-illness analysis. What room in health economics? Health Policy. 2006;77(1):51-63.

31. Base de datos el programa de comparación internacional. Factor de conversión de PPA, PIB (UMN por \$ a precios internacionales). Tasa de cambio oficial (UMN por US\$, promedio para un periodo). EE. UU.: Banco Mundial; 2018.

32. Fang X, Brown DS, Florence CS, Mercy JA. The economic burden of child maltreatment in the United States and implications for prevention. Child Abuse Negl. 2012;36:156-165.

33. Boop S, Axente M, Weatherford B, Klimo P. Abusive head trauma: an epidemiological and cost analysis. J Neurosurg Pediatr. 2016;18:542-549.

34. Friedman J, Reed P, Sharplin P, Kelly P. Primary prevention of pediatric abusive head trauma: a cost audit and cost-utility analysis. Child Abuse Negl. 2012;3:760-770.

35. Dias M, Smith K, DeGuehery K, Mazur P, Li V, Shaffer ML. Preventing abusive head trauma among infants and young children: a hospital-based, parent education program. Pediatrics. 2005;115:e470-e477.

36. Fujiwara T. Effectiveness of public health practices against shaken baby syndrome/abusive head trauma in Japan. Public Health. 2015;129:475-482.

37. Ettaro L, Berger RP, Songer T. Abusive head trauma in young children: characteristics and medical charges in a hospitalized population. Child Abuse Negl. 2004;28:1099-1111.

38. Hartunian NS, Smart CN, Thompson MS. The incidence and economic costs of cancer, motor vehicle injuries, coronary heart disease, and stroke: a comparative analysis. Am J Public Health. 1980;70:1249-1260.

39. Sagastuy B. Retos de los hospitales en el marco de la salud universal y el desarrollo sostenible. En: Fajardo-Ortiz G. Nuevas fronteras en el pensamiento y práctica de la administración hospitalaria. México: Academia Nacional de Medicina/Editorial Intersistemas; 2018.

40. Augustovski F, García S, Pichon-Riviere A. Estándares consolidados de reporte de evaluaciones económicas sanitarias: versión en español de la lista de comprobación CHEERS. Value Health Reg Issues. 2013;16:231-250. 